\title{
Visual and Auditory Stimuli Response, Measured by Emotiv Insight Headset
}

\author{
Martina Zabcikova ${ }^{1, *}$ \\ ${ }^{1}$ TBU in Zlin, Faculty of Applied Informatics, Department of Informatics and Artificial Intelligence, 76005 Zlin, Czech Republic
}

\begin{abstract}
Brain-Computer Interface $(\mathrm{BCI})$ is a very attractive area and common trend worldwide. The electroencephalographic (EEG) technology is being used by $\mathrm{BCI}$ systems that process brain signals through computer algorithms. EEG-based BCI has become an important tool for real-time analysis of brain activity. This article examines the usability and quality of the signals recorded using the Emotiv device. To examine brain activity, visual and auditory stimuli were presented to the subjects and responses was observed using wireless noninvasive EEG device Emotiv Insight. To measure signals the free version of software Emotiv Xavier ControlPanel was used. The results demonstrated that the Emotiv Insight device is suitable for entertainment.
\end{abstract}

\section{Introduction}

The BCI can be defined as a system that translates the brain activity patterns of a user into messages or commands for an interactive application. This activity is measured and processed by the system. The BCI user's brain activity is measured using electroencephalography (EEG) here. [1] EEG is a record of changes in neuronal polarization and neuroglia in the CNS (central nervous system) during the time. Surface electrodes detect changes in neuronal polarization. It is about bioelectric manifestations of brain processes and responses to external stimuli (visual and auditory). Visual stimuli are reactions to stroboscope flash, chessboard, and images. Auditory stimuli are reactions to sounds, tones, and music. Detections can be categorized into cognitive, expressive and affective. Here affective detections are used. Affective input signals are signals sent by the brain based on emotion or meditation. A method is provided for individuals to interact with a computer via emotions and without need to move.

Advances in brain science and computer technology in the past decade have led to significant developments in BCI. Recent technological advances such as wireless recording, low-cost amplifiers, real-time temporal resolution, machine learning analysis, and advanced signal analysis methodology, showed that the EEG-based BCI systems are more accessible to researchers in many scientific domains. The basic division of BCI systems is invasive and non-invasive. Currently, there is a high level of interest in noninvasive BCI technology. The portable EEG headset helps to understand the brain dynamics underlying integration of perceptual functions of the brain in different scenarios. Here wearable and wireless EEG headset Emotiv Insight is used. [2]

Currently, plenty of BCI applications in different research sectors already exist (medicine, communication and control, entertainment, rehabilitation, assistive technology, training, and other). [3]

BCI connects the outside world, in real time, like biological communication system. It facilitates the communication link from the brain to the external world by converting brain thoughts into control commands to control the external devices (robotic arm, wheelchair, drones, neuroprosthesis, exoskeletons, keyboard mouse, playing games, etc.). [4]

In this article, to measure brain activity noninvasive EEG-based BCI device Emotiv Insight is used. Emotiv is one of the $\mathrm{BCI}$ distributers to public users. The electrodes are placed on the surface of the skull. To examine brain activity, is used the record of brain activity responding to visual and auditory stimuli. Emotiv Insight is mobile wireless neuroheadset. This device has EEG sensors that detect neurological activity. The data can be transferred to a computer or smartphone via Bluetooth or USB. To measure signal quality Emotiv Xavier ControlPanel software is used.

\section{Emotiv Insight}

Emotiv Insight is designed for everyday use with advanced electronics that produce clean and robust signals, uses five channel EEG that senses brain activity. Semi-dry polymer sensors are easy to use and clean. Minimal setup time is only about two minutes. This

${ }^{*}$ Corresponding author: zabcikova@utb.cz 
device works wirelessly. You may connect your computer or mobile phone. This device lasts for up to nine hours. To detect head movements nine-axis motion sensors are available. [5]

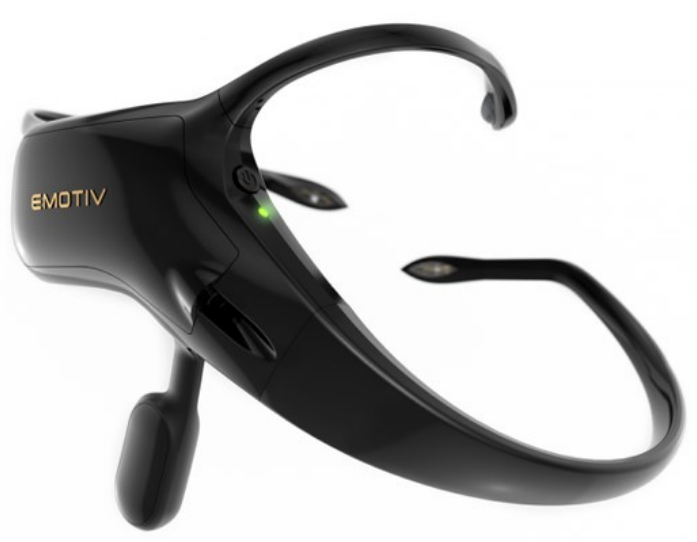

Fig. 1 Headset Emotiv Insight. [5]

There are five EEG sensors to sense brain activity and two CMS/DRL references (left/right mastoid process alternative). CMS (Common Mode Sense) is an active electrode and DRL (Driven Right Leg) is a passive electrode. [5]

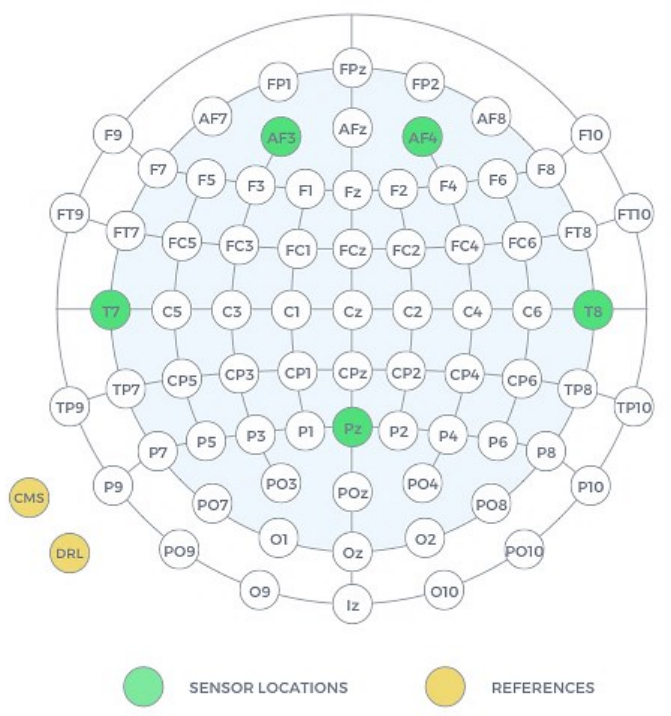

Fig. 2 Locations of seven EEG sensors. [5]

Green sensors are located in the frontal, temporal and parietal part of the head. The yellow sensors are the basic electrode connected to the reference electrode.

\section{Software Emotiv Xavier ControlPanel}

Free software Emotiv Xavier ControlPanel is used to measure the signal. Raw EEG, mental commands, performance metrics, and facial expressions detections are available. In this article, there are affective performance metrics including interest, engagement, stress, relaxation, excitement, and focus.

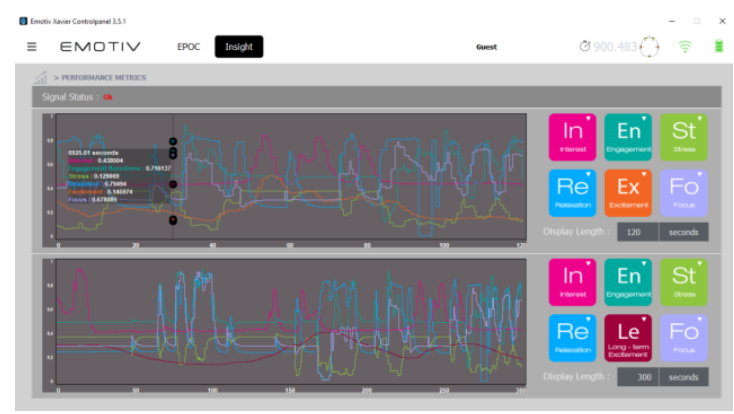

Fig. 3 Software Emotiv Xavier ControlPanel - performance metrics.

The individual performance metrics are distinguished by colors. We can see the signal status at the top of the picture. The signals can be either high quality or not available as you can see in the picture. We can also look at the exact value of each metric.

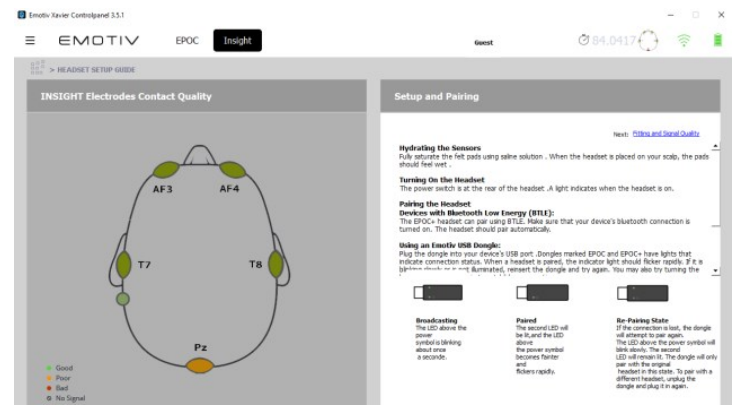

Fig. 4 Software Emotiv Xavier ControlPanel - Insight electrodes contact quality, setup and pairing.

The setup, pairing and signal quality can be checked in Fig. 4. We can see that the Insight device is currently connected and how long it took to setup the device.

\section{Experimental part}

\subsection{Visual stimuli}

Here is used the record of brain activity responding to visual stimuli. For this measurement, a presentation of various images was made. The presentation includes a sequence of pictures of cute animals, funny dog, 
hedgehog in a mug, starved kids, love, cute bunny, scary house, sea, spider, and nature in spring. The total length of the presentation is sixty seconds.

\subsection{Auditory stimuli}

In this part, is used the record of brain activity responding to auditory stimuli. There were six different types of sounds and music. The selection included relaxing music, funny music, death metal, fairy tale, pop music, and horror sounds. The total length of the presentation is one hundred and twenty seconds.

\subsection{Emotiv Insight}

The first step is to attach the rear arm and sensors. The second step is to charge the headset. The third step is to turn on the headset. Here Bluetooth is used for the connection. The last step is adjusting the headset with good contact quality.

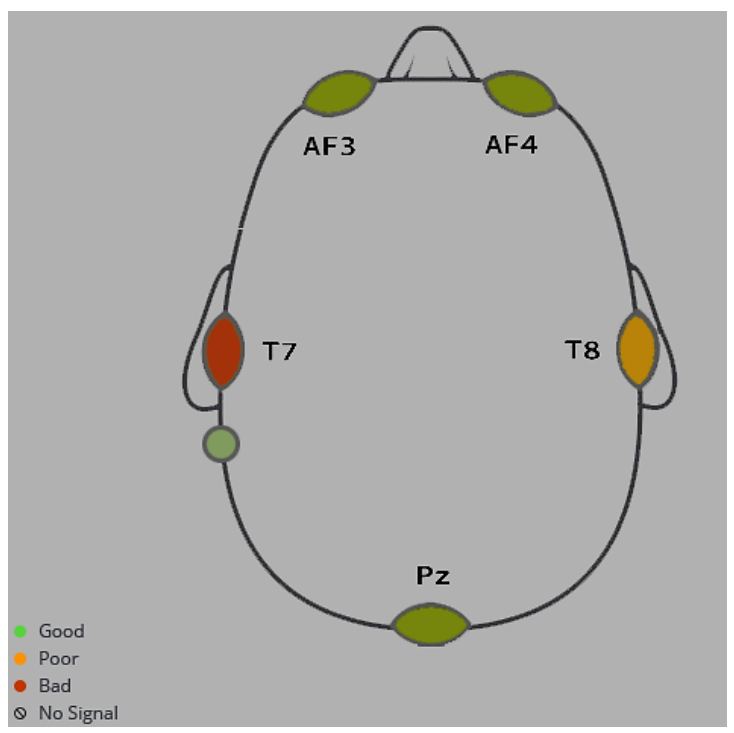

Fig. 5 Emotiv Insight - bad electrodes contact quality.

To achieve good signal quality for all sensors, Emotiv Insight Fluid can be used.

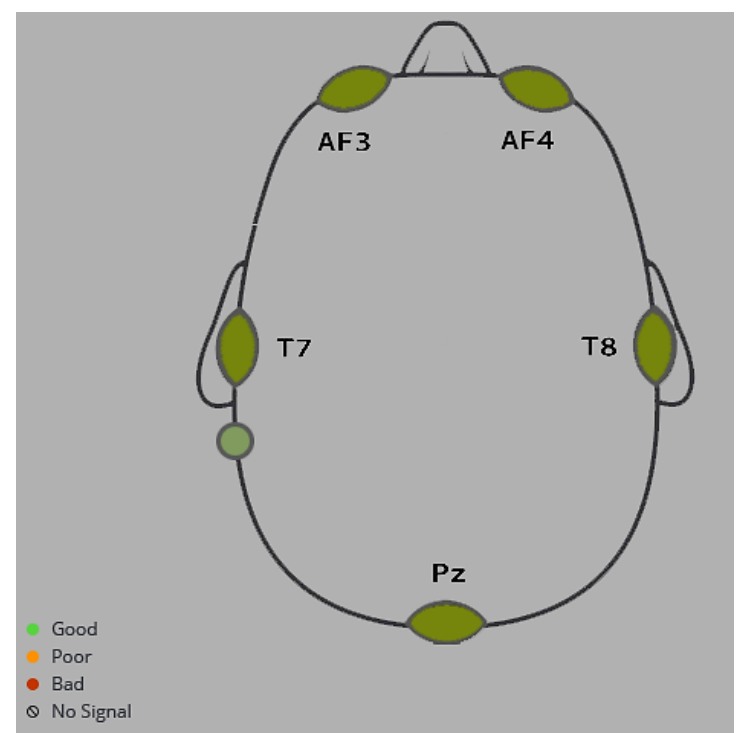

Fig. 6 Emotiv Insight - good electrodes contact quality.

\subsubsection{Visual stimuli - participant 1}

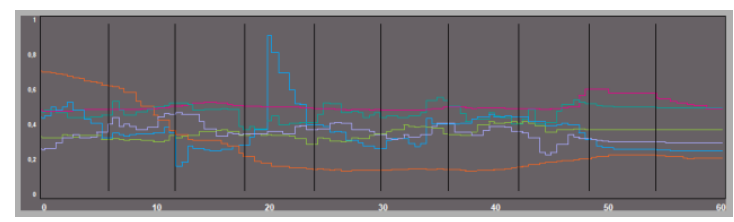

Fig. 7 Participant 1 - brain signals measured by Insight headset responding to visual stimuli.

The level of interest was almost identical throughout the measurement, except for an increase in the image of the sea. The level of engagement for pictures of love, cute bunny, and sea, has increased slightly and decreased in the picture of hedgehog in a mug. The level of stress remained steady for the whole time. The highest changes can be seen in relaxation. Excitement level fell down in pictures of funny dog and hedgehog in a mug. We can also see that the focus was jumping significantly in every change. A slight increase was observed in the picture of cute animals and funny dog, and from the other side, decrement was in the picture of the hedgehog in a mug and sea. 


\subsubsection{Visual stimuli - participant 2}

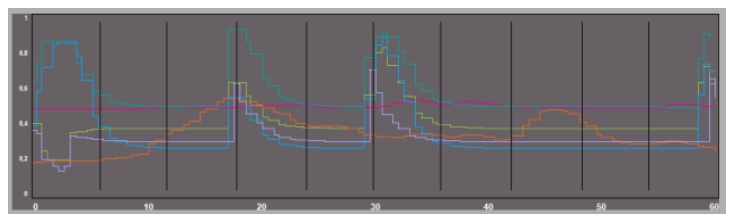

Fig. 8 Participant 2 - brain signals measured by Insight headset responding to visual stimuli.

We can see on the graph that in the individual transitions between images are big changes, but only for some images. The interest is almost the same except for small fluctuations during the measurement. We can see a huge increase in engagement and relaxation in the picture of cute animals, starved kids, cute bunny and nature in the spring. We can see a jump in stress in images of starved kids and a cute bunny. An increased excitement can be seen in the picture of hedgehog in a mug and sea. The rise of focus can be observed in the pictures of starved kids, cute bunny and nature in the spring, while the decrease can be seen in the first picture of a cute animal. We can see that some signals are a bit inaccurate here and significant reactions are only on some images.

\subsubsection{Auditory stimuli - participant 1}

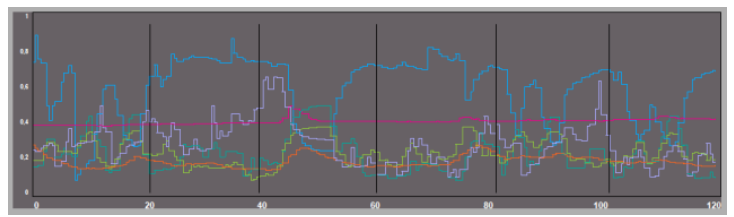

Fig. 9 Participant 1 - brain signals measured by Insight headset responding to auditory stimuli.

On the graph, we can see huge fluctuations in individual types of music. An increase in interest can be observed while listening to death metal. The highest increase in engagement and stress can be seen while listening to death metal. The biggest fluctuations happened within the relaxation. The large increase in excitement can be seen while listening to death metal and fairy tale. High values in focus we see while listening to death metal and pop music.

\subsubsection{Auditory stimuli - participant 2}

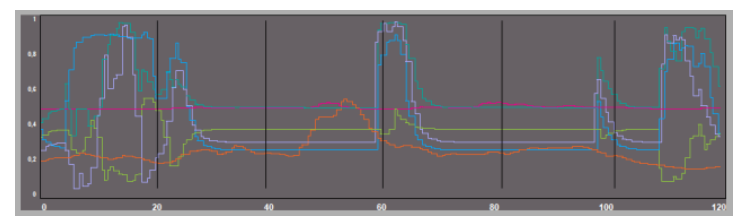

Fig. 10 Participant 2 - brain signals measured by Insight headset responding to auditory stimuli.

We can see huge fluctuations in individual types of music. The interest level remained steady except for small fluctuations throughout the measurement. We can see that the engagement, relaxation, and focus increased individually according to the popular genre of the participant. We can also see a huge increase in relaxation while listening to relaxing music. The stress level decreased most while listening to relaxation music and increased most for horror sounds. The highest increase in excitement was while listening to death metal.

\section{Discussion}

$\mathrm{BCI}$ is defined as a combination of hardware and software that allows brain activities to control external devices or even computers. The objective is to help severely disabled people to live their life as regular people as much as possible. However, this area can also serve healthy people. The field of $\mathrm{BCI}$ is one of the important fields that deals with brain activities. It is expected that BCI applications will have a great effect on our daily life. In the last decade, technological advances in BCIs permit EEG acquisition by means of wireless, mobile, dry, wearable, and low-cost EEG headsets, with new potential daily-life applications. [6]

$\mathrm{BCI}$ can be used for many purposes in many areas, especially in the field of robotic and rehabilitation. One of the steps to optimize the BCI's capabilities, it should be used together with other up-to-date technologies (called hybrid BCI). [7] In another study was explored EEG headset device, Emotiv Insight, for its applicability in cognitive research. The conclusion was that the features and usability of the EEG headset are according to the research requirements. [8] Emotiv Insight is a very lowcost consumer-grade EEG device. 


\section{Conclusion}

This study discusses the quality of the signals and the usability recorded using Emotiv Insight device. The experimental evaluation of the devices was performed with two participants. It was processed by four measurements. Measurement of both first and second participant on visual and audio stimuli was performed by Insight headset. The first participant was a man with short hair. Setup time was about two minutes. The second participant was a woman with long and dense hair. The device Insight was ready for use approximately after five minutes. Insight headset can measure all emotional states, despite the bad signal, so the measurement was not so accurate. Emotiv Insight looks visually good and there is quick setup. Nevertheless, on the other hand, the helmet is quite uncomfortable and pushes. Due to it is not recommended for medical or scientific purposes.

The values of individual participants to various stimuli are very different. It may depend on what the participant preferences. Eventually, this study showed that the Insight is more suitable for entertainment.

This work was supported by IGA (Internal Grant Agency) of Tomas Bata University in Zlin under the project No. IGA/FAI/2019/005.

\section{References}

1. F. Lotte, L. Bougrain, A. Cichocki, M. Clerc, M. Congedo, A. Rakotomamonjy, F. Yger, J. of Neur. Eng. 15(3) (2018)

2. R. Abiri, S. Borhani, E.W. Sellers, Y. Jiang, X. Zhao, J. of Neur. Eng. 16(1) (2019)

3. Ch.S. Nam, A. Nijholt, F. Lotte, Brain-computer interfaces handbook: technological and theoretical advances, Boca Raton: Tayl. \& Franc., CRC Press (2018)

4. L. Junwei, S. Ramkumar, G. Emayavaramban, D.F. Vinod, M. Thilagaraj, V. Muneeswaran et al., IEEE Access, 7 (2019)

5. Emotiv: Emotiv Insight Brainwear 5 Channel Wireless EEG Headset [online]. Available on: https://www.emotiv.com/insight/ (2019)

6. J. Minguillon, M.A. Lopez-Gordo, F. Pelayo, Biom. Sig. Proc. and Con. 31 (2017)

7. R. Ahad, K.A.A. Rahman, M.Z. Mustaffa, N. Fuad, M.K.I. Ahmad, IEEE-EMBS IECBES (2018)

8. M.S. Ijjada, H. Thapliyal, A. Caban-Holt, H.R. Arabnia. 2015 CSCI (2015) 